\title{
Disaggregating Non-Volatile Memory for Throughput-Oriented Genomics Workloads
}

\author{
Aaron Call ${ }^{1,2}$, Jordà Polo ${ }^{1}$, David Carrera ${ }^{1,2}$, Francesc Guim ${ }^{3}$, Sujoy Sen ${ }^{3}$ \\ 1 Barcelona Supercomputing Center (BSC) \\ \{aaron.call, jorda.polo, david. carrera\}@bsc.es \\ 2 Universitat Politècnica de Catalunya (UPC) \\ 3 Intel Corporation \\ $\{$ francesc.guim, sujoy.sen\}@intel.com
}

\begin{abstract}
Massive exploitation of next-generation sequencing technologies requires dealing with both: huge amounts of data and complex bioinformatics pipelines. Computing architectures have evolved to deal with these problems, enabling approaches that were unfeasible years ago: accelerators and Non-Volatile Memories (NVM) are becoming widely used to enhance the most demanding workloads. However, bioinformatics workloads are usually part of bigger pipelines with different and dynamic needs in terms of resources. The introduction of Software Defined Infrastructures (SDI) for data centers provides roots to dramatically increase the efficiency in the management of infrastructures. SDI enables new ways to structure hardware resources through disaggregation, and provides new hardware composability and sharing mechanisms to deploy workloads in more flexible ways. In this paper we study a state-of-theart genomics application, SMUFIN, aiming to address the challenges of future HPC facilities.
\end{abstract}

Keywords: Genomics, Disaggregation, Composability, NVM, NVMeOF, Characterization, Orchestration

\section{Introduction}

The genetic basis of disease is increasingly becoming more accessible thanks to the emergence of Next Generation Sequencing platforms, which have extremely reduced the costs and increased the throughput of genomic sequencing. For the first time in history, personalized medicine is close to becoming a reality through the analysis of each patient's genome. Genomic variations, between patients or among cells of the same patient, have been identified to be the direct cause, or a predisposition to genetic diseases: from single nucleotide variants to structural variants, which can correspond to deletions, insertions, inversions, translocations and copy number variations, ranging from a few nucleotides to large genomic regions. The exploitation of genomic sequencing should involve the accurate identification of all kinds of variants, in order to derive a correct diagnosis and to select the best therapy. For clinical purposes, it is important that this computational 
process be carried out within an effective timeframe. But a simple sequencing experiment typically yields thousands of millions of reads per genome, which have to be stored and processed. As a consequence, the analysis of genomes with diagnostic and therapeutic purposes is still a great challenge, both in the design of efficient algorithms and at the level of computing performance.

The field of computational genomics is quickly evolving in a continuous seek for more accurate results, but also looking for improvements in terms of performance and cost-efficiency. In parallel, computing architectures have also evolved, enabling approaches that were unfeasible only years ago. The use of Non-Volatile Memories (NVM) and accelerators has been widely adopted for all kinds of workloads with the introduction of NVMe cards, GPUs, and FPGAs for some of the most demanding computing challenges. Genomics workloads today have a larger variety of requirements related to the compute platforms they run in. Workloads are tuned to work optimally on specific configurations of compute, memory, and storage. On top of that, current genomics workloads and pipelines tend to be composed of multiple phases with different behaviors and resource requirements.

One such example in the context of variant calling is SMUFIN [15], a state-ofthe-art method that performs a direct comparison of normal and tumor genomic samples from the same patient without the need of a reference genome, leading to more comprehensive results. In its original implementation, published in Nature [15] in 2014, this novel approach required significant amounts of resources in a supercomputing facility. Since then, it has been optimized and adapted to scale up and make the most of Non-Volatile Memory [1.

Beyond Non-Volatile Memories and accelerators, new technological advances currently under development, such as Software Defined Infrastructures, are dramatically changing the data center landscape. One of the key features of Software Defined Infrastructures is disaggregation, which allows dynamically attaching and detaching resources from physical nodes with just a software operation, removing the constraints of getting hardware components statically confined to servers. This paper takes a modern genomics workload, SMUFIN, evaluates disaggregation mechanisms when running it, and describes how characterization can be used to guide the orchestration of a genomics pipeline.

The rest of the paper is structured as follows. Section 2 provides an overview of the foundations of SMUFIN, the variant-calling method studied in this paper. Section 3 introduces resource disaggregation and the technology used to implement it. Next, Section 4 characterizes disaggregation mechanisms using SMUFIN. Section 5 shows how characterization can be used to guide orchestration. And finally, Section 6 discussed related work and Section 7 concludes.

\section{SMUFIN: A Throughput-oriented Genomics Workload}

Most currently available methods for detecting genomic variations rely on an initial step that involves aligning sequence reads to a reference genome generally using Burrows-Wheeler transform [12, which has an impact not only on performance, but also on the accuracy of results. First, tumoral reads that carry 
variation may be harder or impossible to align against a reference genome. Second, the use of references also leads to interference with millions of inherited (germline) variants that affect the actual identification of somatic changes, consequently decreasing the final reliability and applicability of the results. The initial alignment also has an impact on subsequent analysis since most methods are tuned to identify only a particular kind or size of mutation 14 .

Alternative methods that don't rely on the initial alignment of sequenced reads against a reference genome have been developed. In particular, the application used in this work is based on SMUFIN [15, a reference-free approach based on a direct comparison between normal and tumoral samples from the same patient. The basic idea behind SMUFIN can be summarized in the following steps: (i) input two sets of nucleic acid reads, normal and tumoral; (ii) build frequency counters of substrings in the input reads; and (iii) compare branches to find imbalances, which are then extracted as candidate positions for variation.

Internally, SMUFIN consists of a set of checkpointable stages that are combined to build fully fledged workloads (Figure 1). These stages can be shaped on computing platforms depending on different criteria, such as availability or cost-effectiveness, allowing executions to be adapted to its environment. Data can be split into one or more partitions, and each one of these partitions can then be placed and distributed as needed: sequentially in a single machine, in parallel in multiple nodes, or even in different hardware depending on the characteristics of the stage.

Data partitioning can be effectively used to adapt executions to a particular level of resources made available to SMUFIN, because it imposes a trade-off between computation and IO. This data partitioning can be achieved by going multiple times through the input data set that corresponds to each stage: Prune, Count, and Filter. In practice, systems with high-end capabilities will not require a high level of partitioning and hence IO, what ends up with scale-up solutions; on the opposite side of the spectrum, lower-end platforms are able to run the algorithm by partitioning data and duplicating IO, leading to scale-out solutions. The goal of each one of the stages is as follows:

- Prune: Discards sequences from the input by generating a bloom filter of kmers that have been observed in the input more than once. Allows lowering memory requirements at the expense of additional computation and IO.

- Count: Builds a frequency table of normal and tumoral k-mers in the input sequences. More specifically, k-mer counters are used to detect imbalances when comparing two samples.

- Filter: Selects k-mers with imbalanced frequencies, which are candidates for variation, while also building indexes of sequences with such k-mers.

- Merge: Reads and combines multiple filter indexes from different partitions into single, unified indexes. Merging indexes only involves simple operations such as concatenation, OR on bitmaps, and appending.

- Group: Matches candidate sequences that belong to the same region. First, selecting reads that meet certain criteria, and then retrieving related reads by looking up those that contain the same imbalanced k-mers. 


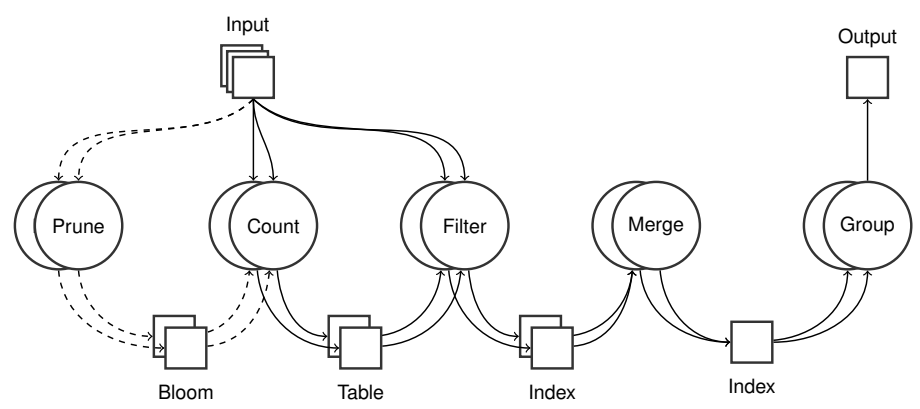

Fig. 1: SMUFIN's variant calling architecture: overview of stages and its data flow

One of the main characteristics of the current version of SMUFIN [1] is its ability to use NVM as memory extension. This can be exploited in two different ways. First, using an NVM optimized Key-Value Store such as RocksDB, and second, using a custom optimized swapping mechanism to flush memory directly to the device. When such memory extensions are available, a maximum size for the data structures is set; once such size is reached, data is flushed to the memory extension while a new empty structure becomes available Generally speaking, bigger sizes are recommended: they help avoid duplicate data, and also lead to higher performance, as writing big chunks to a Non-Volatile Memory allows to exploit internal parallelism typical of flash drives [2].

SMUFIN's performance greatly benefits from NVM, as shown in Figure 2 . which compares an execution in 16 machines in a supercomputing facility (left) and a scale-up execution in a single node with NVM enabled (right). The latter leads to faster executions and lower power consumption. NVM can be leveraged in some way in most SMUFIN stages, and the experiments performed in this paper are focused on Merge using the RocksDB-based implementation, which is one of the most IO intensive of the pipeline. However, other stages have similar characteristics and the same techniques can be used elsewhere.

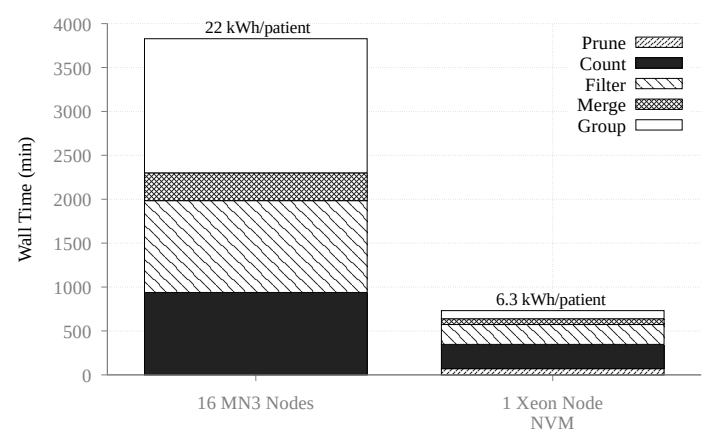

Fig. 2: Aggregate CPU time of a SMUFIN execution running in 16 MareNostrum nodes and in 1 Xeon-based node with NVM. Power consumption per execution (one patient) shown for reference.

\section{Resource Disaggregation}

Traditional data centers usually contain homogeneous and heterogeneous compute platforms (also referred to as computing nodes or servers). These platforms 
are statically composed by computing, memory, storage, fabric, and/or accelerator components, and they are usually stored in racks. However, in the last few years there has been a trend towards new technologies that allow disaggregating resources over the network, increasing flexibility and easying the management of such data centers.

This paper analyzes the use of one of those new technologies: NVMe Over Fabrics (NVMeOF). First off, NVMe 17 is an interface specification for accessing direct-attached NVM via a regular PCI Express bus. On the other hand NVMeOF 4 is an emerging network protocol used to communicate nodes with NVMe devices over a networking fabric. The architecture of NVMeOF allows scaling to large numbers of devices, and supports a range of different network

fabrics, usually through Remote Direct Memory Access (RDMA) so as to eliminate middle software layers and provide very low latency.

Disaggregating NVMe over the network with NVMeOF allows new mechanisms to scale-up and improve efficiency of genomics workloads:

Resource Sharing. As workloads perceive remote NVMe as physically attached to their compute nodes, those can be partitioned, and each one of these partitions can then be exposed to the computational nodes as an exclusive resource. This translates into workload-unaware resource sharing, which in turn can lead to improved resource efficiency by maximizing usage.

Resource Composition. Certain resources can be aggregated and exposed as a single, physically attached resource. Instead of accessing individual units, accessing combined resources enables increased capacities that can lead to improved performance. For instance, two SSD disks with a bandwidth of $2 \mathrm{~GB} / \mathrm{s}$ each can be composed and exposed as a single one with twice as much capacity and bandwidth, providing a total of $4 \mathrm{~GB} / \mathrm{s}$.

\section{Characterizing Resource Disaggregation on SMUFIN}

In a continuous need to deal with increasingly larger amounts of data, genomics workloads are quickly adapting, and NVM technologies have become widely used as a key component in the memory-storage hierarchy. This Section explores how disaggregating NVM might have an impact on genomics workloads, and in particular SMUFIN. As part of the evaluation, resource sharing and composition are analyzed using NVMeOF in an attempt to scale-up and shape the performance of the workload.

\subsection{Experimental Environment}

The experiments are conducted in an environment as depicted in figure 3 . The NVMe drives are used by SMUFIN as a memory extension over fabric to store temporary data structures required to accelerate the computation. As the drives are dual-controller, two NVMe devices - of half its physical size - are exposed by the system for each physical device. In order to expose a single NVMe consisting 


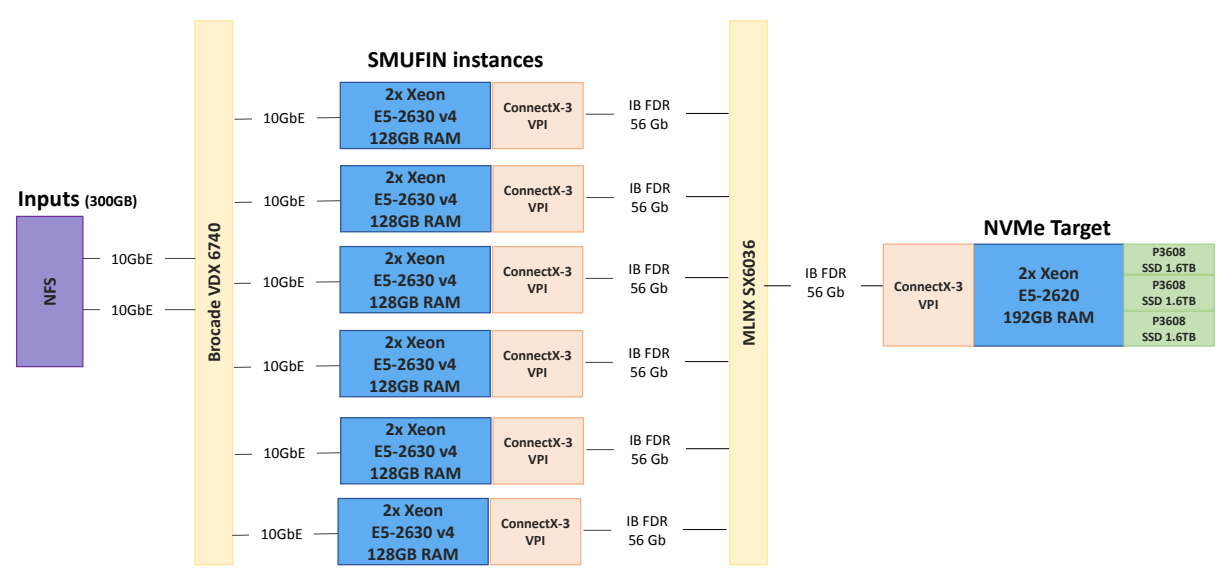

Fig. 3: Experiments environment

of its two controllers, or to unify several NVMe devices, Intel Rapid Storage Technology [9] (RST) is used. RST composes a RAID0 of the controllers which becomes exposed over fabric as a single NVMe card. Mellanox OFED 4.0-2.0.0.1 drivers were used for the InfiniBand HCA adapters. The drivers included modules for NVMe over fabrics as well, both the target and the client. Kernel 4.8.0-39 was used under Ubuntu server 16.10 operating system in all nodes.

We use SMUFIN on its merge stage, as explained in section 2. In the following evaluations each SMUFIN instance reads and processes a sample DNA input (+300GB) from a NFS shared storage, while the shared NVMe devices are used as memory extension for temporary data and final output. SMUFIN has been implemented to maximize sequential writes to the devices, and this behavior has been verified by analyzing its access pattern. A block trace sample of requested blocks to the device was generated using Linux's blktrace, and the trace was then fed to the algorithm provided by [3] to calculate the percentage of sequential write accesses. This method identified $88 \%$ of sequential writes after adapting the algorithm to consider accesses in which the final address matched the initial address of many immediately following requests, thus accounting for file appends.

\subsection{Direct-Attached Storage vs NVMe over Fabrics}

The performance of NVMeOF has been studied in the literature [7, and found not to show any significant degradation when compared to local directly-attached storage (DAS). Additionally, in this section we perform our own experiments running up to 3 instances of SMUFIN in the same node: against a directlyattached NVMe device and against NVMeOF. Each instance processes the same dataset, generating $\approx 150 \mathrm{~GB}$, with an average use of bandwidth of $477 \mathrm{MB} / \mathrm{s}$ per SMUFIN instance. The NVMe device is capable of handling $2 \mathrm{~GB} / \mathrm{s}$ bandwidth under sequential write pattern, as is the SMUFIN scenario. Figure 4 shows average execution time and deviation after repeating the executions six times. As 
(a) DAS

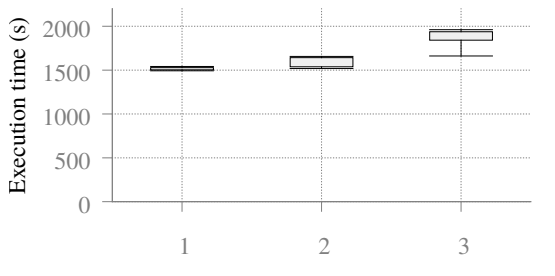

(b) NVMeOF

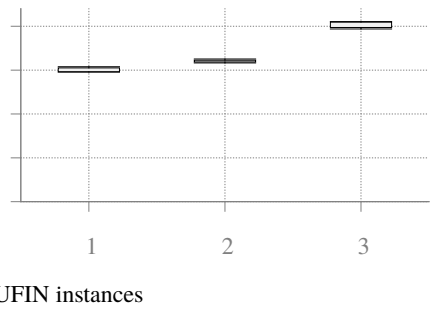

Fig. 4: Boxplot of execution time of Direct-Attached Storage (DAS) and NVMeOF when running 1x, $2 \mathrm{x}$ and $3 \mathrm{x}$ SMUFIN instances on the same node

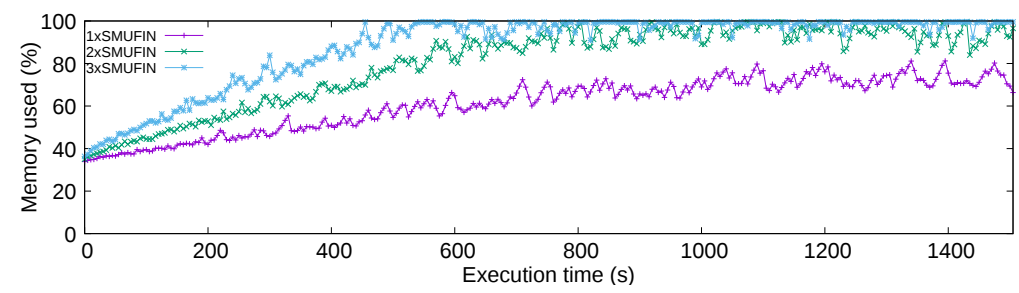

Fig. 5: Memory usage on $1 \mathrm{x}, 2 \mathrm{x}, 3 \mathrm{xSMUFIN}$ scenarios using Direct-Attached NVMe on a period of 1500 seconds

it can be observed, when running one and two instances on local storage ( 4 a) there is no performance degradation when disaggregating NVMe over fabrics ( $4 \mathrm{~b})$. However, when running three concurrent instances there is a significant degradation of $6 \%$ when using NVMeOF.

On the other hand there is a certain performance degradation scaling up to three instances in both scenarios. Analyzing this behavior, up to two instances, the host's memory can handle all the intermediate data generated by SMUFIN and the NVMe becomes only used to output final data. However, with three instances the memory becomes a bottleneck and intermediate data not fitting in memory gets flushed to the NVMe device more frequently. Is in this scenario when degradation is observed and performance comparison against $\mathrm{NVMeOF}$ is worse. Figure 5 depicts memory usage on the three scenarios (1, 2 and 3 SMUFIN on the same node, directly-attached) over a period of 1500 seconds, evidencing the memory bottleneck.

\subsection{Resource Sharing And Composability}

When multiple workloads share resources and hence compete for its usage, their execution time compared to a dedicated execution in isolation degrades when a threshold is reached, as shown in previous section. In this section we explore if degradation still occurs when running up to six concurrent instances, all of them using partitions from the same set of NVMe devices and running on separate nodes to avoid the aforementioned interferences.

Figure 6a represents the box plot of individual execution times under different configurations, along with its quartiles, median, and standard deviation. 
(a) $1 \times \mathrm{NVMeOF}$

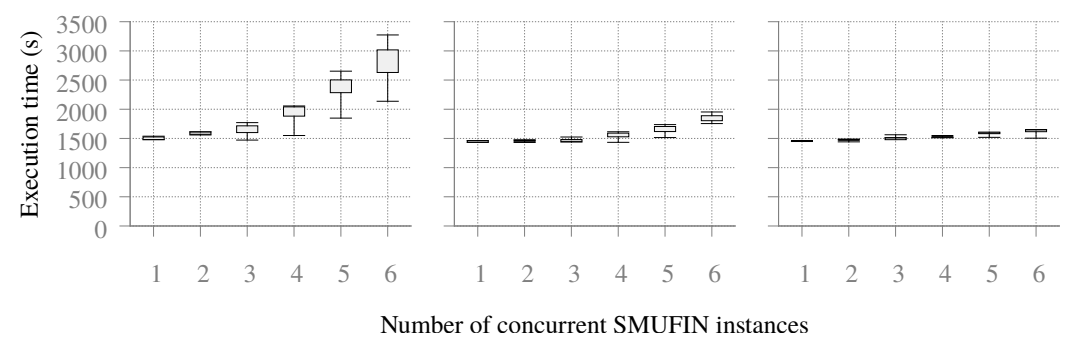

Fig. 6: Boxplots showing how execution time evolves when running multiple SMUFIN instances: sharing a single device (1xNVMe), or sharing on composed nodes (2xNVMe, $\left.3 \times x_{V M M}\right)$

In (a) only one NVMe SSD is used. It can be observed running three instances separately against a single device do not degrade as significantly as running under the same node. However, performance degradation is still experienced when certain resource sharing threshold is reached.

When disaggregating NVMe over fabrics we can benefit of composing several NVMe devices and expose them as a single one. Under composition, profiling data shows that the Intel driver balances the bandwidth evenly through all composed devices. It is also observed that provided bandwidth scales linearly with the number of devices, hence under 2 and 3-compositions $4 \mathrm{~GB} / \mathrm{s}$ and $6 \mathrm{~GB} / \mathrm{s}$ of sequential write speed can be reached respectively (each individual drive provides $2 \mathrm{~GB} / \mathrm{s}$ ). Through composition, performance degradation can be mitigated. Compositions of two and three NVMe SSD exposed as a single target to clients increases the bare-performance, as a composition multiplies the total available bandwidth. The evolution of execution time respect composition level is presented in Figures 6 $\mathrm{b}$ and 6r. In the 2-composition scenario, up to 3 sharing workloads obtain the same performance as if running alone in a single NVMe. The level of concurrency can be increased without introducing significant degradation using a composition of $3 \mathrm{NVMe}$, being able to have six sharing workloads with a similar performance as when running alone in a single device. Thus, workloads indeed benefit of resource composition. However, in all scenarios performance degradation still occurs on reaching a certain threshold, larger as more devices are used. Under 2-NVMe compositions it is at four workloads, whereas on the 3-composition the tendency is observed at six instances threshold.

\subsection{Bandwidth}

We observed performance degradation when a certain sharing ratio of resources is reached. Despite composition increases this threshold, degradation still occurs regardless of composition. As the memory bottleneck was removed and cannot be found on the network bandwidth, we analyze the target NVMe bandwidth.

Figures $7 \mathrm{a}$ and $7 \mathrm{~b}$ show the NVMe bandwidth over time for experiments running up to four concurrent SMUFIN instances in the single-resource and the 2-composed resource configuration. The solid horizontal lines indicate the maximum bandwidth for sequential write that the resources can provide $(2 \mathrm{~GB} / \mathrm{s}$ in single-resource configuration, $4 \mathrm{~GB} / \mathrm{s}$ for the composed scenario). 


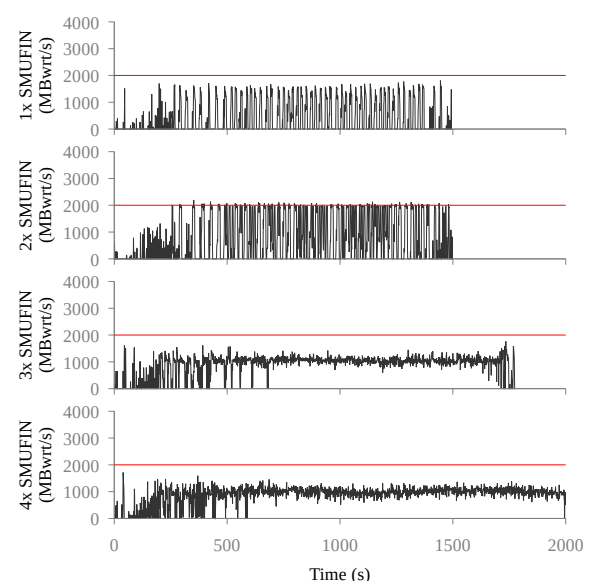

(a) $1 \mathrm{x}$ NVMe
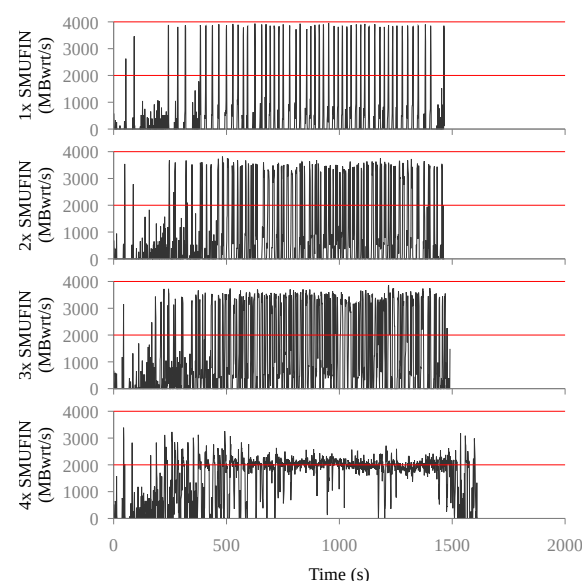

(b) $2 \mathrm{x}$ NVMe

Fig. 7: Bandwidth measured from the NVMe pool server for $1 \mathrm{x}, 2 \mathrm{x}, 3 \mathrm{x}$ and $4 \mathrm{x}$ instances of SMUFIN

From the figures it can be appreciated, on one hand, that resource composition scales linearly, doubling the maximum available bandwidth of a single resource. In both scenarios, two important characteristics can be noticed as more concurrent instances are included in the experiment: (1) the bandwidth observed from the NVMe perspective is steadier; (2) the bandwidth that the NVMe device is capable of delivering is reduced as more concurrent instances are added. Running a single instance, the full bandwidth of the combined NVMe can be used with bursts at the maximum $4 \mathrm{~GB} / \mathrm{s}$. However as more concurrent executions are added these bursts make use of less bandwidth until reaching saturation levels, decreasing significantly.

\section{Towards Efficient Orchestration of Shared and Composed Resources}

Previous sections have shown how NVMe disaggregation provides new ways to use resources through resource sharing and composition. However, its behavior is not obvious a priori: heavy resource sharing may have a negative impact on performance, whereas composition may help increase sharing ratios without degradation. Therefore, deciding whether to compose a resource or to share it among many workloads is not trivial decision. With the help of workload characterization, platform orchestrator will be able to make more informed and smarter decisions.

In Figure 8 we present different orchestration policies that could be managed with our data. The figure shows our cluster running five concurrent instances of SMUFIN, and three different resource allocation strategies for the instances: a) sharing a single device, b) sharing two NVMe devices, and c) one instancededicated device and the remaining four instances on a shared NVM device. 


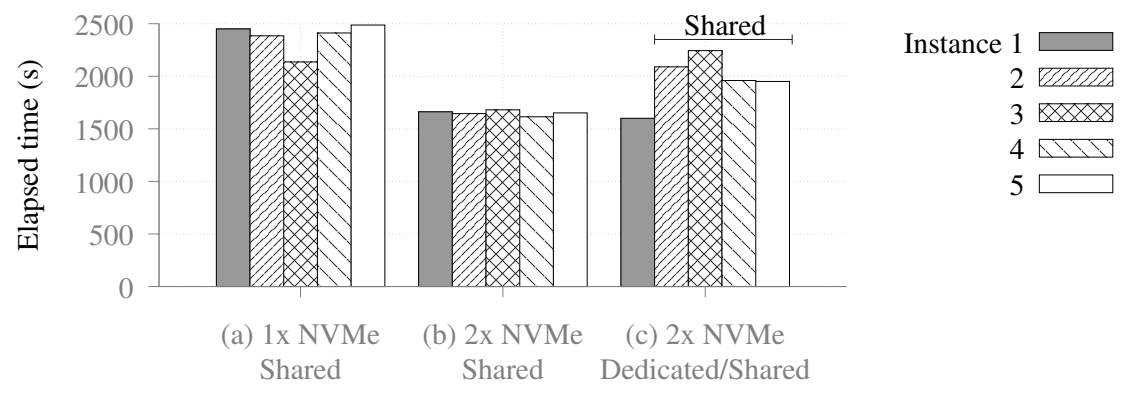

Fig. 8: Execution time of 5 SMUFIN instances under different scenarios

This example was run under the same setup as in section 4 . When the SMUFIN instances use two composed devices (b) it leads to faster executions times than using a single device (a). However, when using a dedicated device to run a single instance and a shared device to run the remaining four (c), the dedicated-device does not grant that instance an improved performance compared to a fully shared scenario using both devices (b). Intuitively it might be believed that just sharing all the resources under composition is the obvious winning strategy. However this approach does not consider arriving workloads might have a time requirement for completion, and upon arrival of those workloads, if the resources are fully occupied serving others the orchestrator will be unable to meet the requirement. Other concerns might be power consumption or total cost of ownership (as more resources, more expensive it becomes to run). Therefore, the strategy to follow must consider the trade-off between execution time and requirements of current and incoming workloads to maximize the granted quality of service, which in the case of genomics might be critical. The work on those policies is out of the scope of this paper and left as future work.

\section{Related work}

Genomics workloads and pipelines in general are a good fit for disaggregation, but prior to this paper applications haven't explored its large-scale explotation. A number of different approaches to parallelize whole genome analysis in HPC systems have been proposed in the literature [16, [10], and [13, but these tend simply adapt existing algorithms without considering or taking complete advantage of next generation computing platforms.

Resource disaggregation is being increasingly studied in the literature. In [6], the authors examine the network requirements for disaggregating resources at rack- and data-center levels. Minimum requirements are measured in terms of network bandwidth and latency. Those requirements must be such so that a given set of applications doesn't experiment any performance degradation when disaggregation memory or other resources over the fabric. 11] implements NVMe disaggregation, but unlike the work presented in this paper, the authors focus on a custom software layer to expose devices instead of using the NVMeOF standard. On the other hand, [18] evaluates the impact of FPGA disaggregation. 
In terms of Software-Defined Infrastructures, Intel Rack Scale [8] is a prototype system that allows dynamic composition of nodes. It fully disaggregates resources in pools, such as CPU, storage, memory, FPGA, GPU, etc. Facebook has engaged with Intel developing its own prototype, the Facebook Disaggregated Rack [5].

\section{Conclusions}

This paper evaluates resource sharing and composition benefits for NVM-centric workloads in the context of disaggregated datacenters. This work takes SMUFIN, a real production workload in the field of Computational Genomics, leveraging remote NVMe devices as memory extension. This paper presents a comprehensive characterization of SMUFIN's resource consumption patterns. It is shown NVMe is utilized in a sequential write pattern. A performance comparison between directly-attached NVMe and NVMeOF is then presented and shown that as long the system's memory is capable of handling SMUFIN instances there is no degradation. To increase concurrency disaggregating over fabrics allows to share the same resource across multiple nodes running instances, as well as the possibility of composition. Thus, through disaggregation we are able to handle more concurrent SMUFIN instances without individual degradation. On the other hand, reaching the resources' sharing ratio limit significantly degrades performance as the utilization of the available bandwidth diminishes, never reaching its maximum. Thus the NVMe becomes the bottleneck. Finally the paper briefly explains how the results of this characterization could be used to implement data-center scheduling policies in order to maximize the efficiency in terms of Quality of Service. Quality of Service could be understood in terms of execution time, so all workloads should be completing its executions within a certain requested time-frame. The work on those policies is left as future work.

\section{Acknowledgment}

This work is partially supported by the European Research Council (ERC) under the EU Horizon 2020 programme (GA 639595), the Spanish Ministry of Economy, Industry and Competitivity (TIN2015-65316-P) and the Generalitat de Catalunya (2014-SGR-1051).

\section{References}

1. Cadenelli, N., Polo, J., Carrera, D.: Accelerating k-mer frequency counting with GPU and Non-Volatile Memory. In: Proceedings of the 19th IEEE International Conference on High Performance Computing and Communications (HPCC). IEEE Computer Society (Dec 2017)

2. Chen, F., Lee, R., Zhang, X.: Essential roles of exploiting internal parallelism of flash memory based solid state drives in high-speed data processing. In: 2011 IEEE 17th International Symposium on High Performance Computer Architecture. pp. 266-277 (Feb 2011) 
3. Ciciani, B., Didona, D., Sanzo, P.D., Palmieri, R., Peluso, S., Quaglia, F., Romano, P.: Automated workload characterization in cloud-based transactional data grids. In: 2012 IEEE 26th International Parallel and Distributed Processing Symposium Workshops PhD Forum. pp. 1525-1533 (May 2012)

4. express, N.: Nvme over fabrics overview. Tech. rep., NVM express (2017), http: //www.nvmexpress.org/wp-content/uploads/nvme_over_fabrics.pdf

5. Facebook, I.: Facebook disaggregated rack. http://goo.gl/6h2Ut (2016)

6. Gao, P.X., Narayan, A., Karandikar, S., Carreira, J., Han, S., Agarwal, R., Ratnasamy, S., Shenker, S.: Network requirements for resource disaggregation. In: Proceedings of the 12th USENIX Conference on Operating Systems Design and Implementation. USENIX Association, Berkely, CA, USA (Nov 2016)

7. Guz, Z., Li, H.H., Shayesteh, A., Balakrishnan, V.: NVMe-Over-Fabrics performance characterization and the path to low-overhead flash disaggregation. In: Proceedings of the 10th ACM International Systems and Storage Conference. pp. 16:1-16:9. SYSTOR '17, ACM, New York, NY, USA (2017)

8. Intel: Intel rack scale design. Tech. Rep. 332937-004, Intel Corporation (aug 2016), http://www . intel.com/content/dam/www/public/us/en/documents/ guides/platform-hardware-design-guide.pdf

9. Intel: Rapid storage. http://www.intel.com/content/www/us/en/support/ technologies/intel-rapid-storage-technology-intel-rst.html (2017)

10. Kawalia, A., Motameny, S., Wonczak, S., Thiele, H., Nieroda, L., Jabbari, K., Borowski, S., Sinha, V., Gunia, W., Lang, U., et al.: Leveraging the power of high performance computing for next generation sequencing data analysis: tricks and twists from a high throughput exome workflow. PloS one 10(5), e0126321 (2015)

11. Klimovic, A., Litz, H., Kozyrakis, C.: Reflex: Remote flash \& local flash. In: Proceedings of the Twenty-Second International Conference on Architectural Support for Programming Languages and Operating Systems. ASPLOS '17 (2017)

12. Li, H., Durbin, R.: Fast and accurate short read alignment with burrows-wheeler transform. Bioinformatics 25(14), 1754-1760 (2009)

13. Li, R., Li, Y., Fang, X., Yang, H., Wang, J., Kristiansen, K., Wang, J.: Snp detection for massively parallel whole-genome resequencing. Genome research 19(6), 1124-1132 (2009)

14. Medvedev, P., Stanciu, M., Brudno, M.: Computational methods for discovering structural variation with next-generation sequencing. Nature methods 6, S13-S20 (2009)

15. Moncunill, V., Gonzalez, S., Beà, S., Andrieux, L.O., Salaverria, I., Royo, C., Martinez, L., Puiggròs, M., Segura-Wang, M., Stütz, A.M., et al.: Comprehensive characterization of complex structural variations in cancer by directly comparing genome sequence reads. Nature biotechnology 32(11), 1106-1112 (2014)

16. Puckelwartz, M.J., Pesce, L.L., Nelakuditi, V., Dellefave-Castillo, L., Golbus, J.R., Day, S.M., Cappola, T.P., Dorn, II, G.W., Foster, I.T., McNally, E.M.: Supercomputing for the parallelization of whole genome analysis. Bioinformatics 30(11), 1508 (2014)

17. Sivashankar, Ramasamy, S.: Design and implementation of Non-Volatile Memory express. In: 2014 International Conference on Recent Trends in Information Technology. Chennai, India (April 2014)

18. Weerasinghe, J., Abel, F., Hagleitner, C., Herkersdorf, A.: Disaggregated FPGAs: Network performance comparison against bare-metal servers, virtual machines and linux containers. In: Proceedings of the 8th IEEE International Conference on Cloud Computing Technology and Science (Dec 2016) 